\title{
ON $P^{2}$-IRREDUCIBLE 3-MANIFOLDS ${ }^{1}$
}

\author{
BY WOLFGANG HEIL
}

Communicated by Morton Curtis, January 16, 1969

In [2] F. Waldhausen proved that two (compact) orientable 3-manifolds which are irreducible, boundary irreducible and sufficiently large, are homeomorphic iff there exists an isomorphism between the fundamental groups which respects the peripheral structure. This is Corollary (6.5) to his Theorem (6.1) in [2]. We extend this theorem to apply to nonorientable 3-manifolds.

Throughout this paper a "manifold" $M$ is a compact 3-manifold. A "surface $F$ in $M$ " is a properly embedded connected 2-manifold in $M$ (i.e. $F \cap \delta M=\delta F$ ).

\section{The main theorem.}

Definition. (1) A manifold $M$ is $P^{2}=$ irreducible iff $M$ is irreducible and does not contain 2 -sided projective planes (properly embedded).

(2) $M$ is boundary irreducible iff $\delta M$ is incompressible (see definition in [2]).

Theorem A. Let $M, N$ be $P^{2}$-irreducible and boundary irreducible. If $M$ is orientable and closed let $M$ be sufficiently large. Let $\pi_{1}(N) \neq 1$ and suppose $f:(N, \delta N) \rightarrow(M, \delta M)$ is a map such that

$$
\operatorname{ker}\left(f_{*}: \pi_{1}(N) \rightarrow \pi_{1}(M)\right)=0 .
$$

Then there exists a homotopy $f_{\gamma}:(N, \delta N) \rightarrow(M, \delta M), \gamma \in I$, of $f=f_{0}$ such that either (a) or (b) holds.

(a) $f_{1}: N \rightarrow M$ is a covering map.

(b) $N$ is a line bundle over a closed surface and $f_{1}(N) \subset \delta M$. $\delta N$.

If $f / \delta N$ is locally homeomorphic, then $f_{\gamma}$ may be chosen constant on

Corollary. Let $M, N$ be $P^{2}$-irreducible and boundary irreducible. If $M$ is orientable and closed let $M$ be sufficiently large. Suppose $\sigma: \pi_{1}(N)$ $\rightarrow \pi_{1}(M)$ is an isomorphism which respects the peripheral structure. Then there exists a map $f: N \rightarrow M$ which induces $\sigma$ and such that either (a) or (b) holds.

(a) $f: N \rightarrow M$ is a homeomorphism.

(b) $M$ is the product line bundle over a closed surface $F$ and $N$ is a twisted line bundle over $F$ and $f:(N, \delta N) \rightarrow(M, \delta M)$ contracts into $\delta M$.

1 These results were obtained while the author was a DAAD-fellow at the University of Illinois in 1967/68. Full details will appear in the author's thesis at Rice University. 
Our proof of the theorem is analogous to the proof of (6.1) in [2]. We will need the following facts.

2. Auxiliary theorems. The analogue of Theorem A for 2-manifolds is

THEOREM 1. Let $f:(G, \delta G) \rightarrow(F, \delta F)$ be a map between two surfaces $G$ and $F$ such that $\operatorname{ker}\left(f_{*}: \pi_{1}(G) \rightarrow \pi_{1}(F)\right)=0$. Let $F \neq P^{2}, \pi_{1}(G) \neq 0$. Then there exists a homotopy $f_{\gamma}:(G, \delta G) \rightarrow(F, \delta F), \gamma \in I$ of $f=f_{0}$ such that either (a) or (b) holds.

(a) $f_{1}: G \rightarrow F$ is a covering.

(b) $G$ is an annulus or moebius band and $f_{1}(G) \subset \delta F$.

If $f / \delta G$ is locally homeomorphic, the homotopy may be chosen constant on $\delta G$.

The proof is analogous to the proof of Theorem A.

Lemma 2. Let $M$ and $N$ be $P^{2}$-irreducible manifolds. Let $F$ be a system of 2-sided incompressible surfaces in $M$ and let $f: N \rightarrow M$ be a map. Then there exists a map $g: N \rightarrow M$, homotopic to $f$, such that $g$ is transverse with respect to $F$ and $g^{-1}(F)$ is a system of 2-sided incompressible surfaces in $N$. If $f / \delta N$ was transverse with respect to $F$ then the homotopy from $f$ to $g$ may be chosen constant on $\delta N$.

The proof is analogous to the proof of Lemma 1 in [3].

THEOREM 3. Let $M$ be $P^{2}$-irreducible. If $M$ is orientable suppose that $\delta M \neq \varnothing$. Then there exists a hierarchy for $M=M_{1}$ i.e. a sequence of manifolds $M_{j}$ and 2-sided incompressible surfaces $F_{j}$ in $M_{j}$,

$$
F_{j} \subset M_{j}, U\left(F_{j}\right) \subset M_{j} ; \quad M_{j+1}=\overline{M_{j}-U\left(F_{j}\right)}, \quad 1 \leqq j \leqq n
$$

$\left(U\left(F_{j}\right)\right.$ is a regular neighborhood in $M_{j}$ ) and each component of $M_{n+1}$ is a ball.

Furthermore every $F_{j}$ can be chosen to be nonseparating in $M_{j}$.

SKETCH OF THE PROOF. Computing the first Betti number $p_{1}$ of $M$ shows that $H_{1}(M)$ is infinite. Therefore there is a nontrivial map $\sigma: \pi_{1}(M) \rightarrow \pi_{1}\left(S^{1}\right)$ and there exists a map $f: M \rightarrow S^{1}$ which induces $\sigma$, since $S^{1}$ is aspherical. By a general position lemma (cf. Lemma 2) the inverse image under $f$ of a point in $S^{1}$ contains a nonseparating 2 -sided surface $F_{1}$ in $M$. By $(\rho)$ and $(\sigma)$-operations, introduced by W. Haken [1], we make $F_{1}$ incompressible and normal with respect to a handle-decomposition [1], [2] of $M$. We do the same thing with

$$
M_{2}=\overline{M-U\left(F_{1}\right)}
$$


and proceed by induction. This process will terminate after a finite number of steps (we have to use the fact that every $F_{j}$ is nonseparating in $M_{j}$ ).

REMARK. If $M$ is orientable then it is easy to show that the image of $H_{1}(\delta M) \rightarrow H_{1}(M)$ is infinite and therefore a map $f: M \rightarrow S^{1}$ may be found such that $f^{-1}$ (point) is a system $F$ of incompressible surfaces with $\delta F \neq \varnothing$. If $M$ is nonorientable then $H_{1}(\delta M) \rightarrow H_{1}(M)$ may be finite as the following example (communicated to the author by J. Hempel) shows: Let $M$ be the solid Klein bottle. If $\delta M$ is generated by $a$ and $b$, where $b^{2} \sim 0$, remove a regular neighborhood of a simple closed curve $a^{\prime} \simeq a^{2}$ in the interior of $M$, and attach the boundary of a solid torus to the resulting boundary such that $a^{\prime}$ is killed. The result is a manifold $M^{\prime}$ which is $P^{2}$-irreducible but $H_{1}\left(\delta M^{\prime}\right) \rightarrow H_{1}\left(M^{\prime}\right)$ is finite.

Proposition 4. Let $F$ be a closed surface, $F \neq S^{2}, P^{2}$. Let $M=F \times I$ be a 2-sheeted cover of a manifold $N$. Then $N$ is homeomorphic to a line bundle over a closed surface.

This may be proved analogous to (4.1) in [2] by constructing a fibering of $M$ which is invariant under the covering translation.

Proposition 5. Let $M$ be $P^{2}$-irreducible and $F \neq F^{1}$ incompressible boundary components. Suppose: For any closed curve $k$ in $F$ some nonnull multiple is homotopic to a curve in $F^{1}$. Then $M$ is homeomorphic to $F \times I$. (Neither $M$ nor $F^{1}$ are assumed to be compact.)

For orientable $M$, this is Lemma 5.1 of [2]. If $M$ is nonorientable then $M^{\prime}$, the 2 -sheeted orientable cover of $M$, satisfies the condition of 5.1 [2], and hence is a product bundle over a closed surface. Then Proposition 5 follows from Proposition 4.

3. Outline of proof of Theorem A. By Theorem 3, choose a hierarchy for $M$. By Lemma 2 deform $f$ such that $f^{-1}\left(F_{j}\right)$ is a system of 2 -sided incompressible nonseparating surfaces in $N$. Furthermore deform $f$ such that $f / f^{-1} U\left(F_{j}\right)$ is a covering map. This can be done for all $F_{j}$ by Theorem 1 provided that no component of $f^{-1}\left(F_{j}\right)$ is a disc or an annulus or moebius band. If this can also be done in the latter case, then case (a) of Theorem A follows. If $f / f^{-1}\left(F_{j}\right)$ is not homotopic to a covering map in case $f^{-1}\left(F_{j}\right)$ is a disc, an annulus or a moebius band then, as in [2], case (b) of Theorem A follows from Propositions 4 and 5.

To prove the corollary note that since $M$ is aspherical and boundary irreducible a map $f:(N, \delta N) \rightarrow(M, \delta M)$ with $f_{*}=\sigma$ can be con- 
structed. If $N$ is not a line bundle, Theorem $\mathrm{A}$ gives a 1-sheeted covering map. If $N$ is a line bundle we prove that $M$ has to be a line bundle, too. Furthermore, looking at the given isomorphism $\sigma$ we may prove case (b) of the corollary.

\section{REFERENCES}

1. W. Haken, Theorie der Normalflaechen, Acta Math. 105 (1961), 245-375.

2. F. Waldhausen, On irreducible 3-manifolds which are sufficiently large, Ann. of Math. (2) 87 (1968), 56-88.

3. - Gruppen mit Zentrum und 3-dimensionale Mannigfaltigkeiten, Topology 6 (1967), 505-517.

Rice University, Houston, Texas 77001 